\title{
Frontières
}

\section{Jouer et danser la tarentelle pour guérir la morsure de la tarentule Réflexions ethnopsychiatriques sur un «culte de la mort »}

\section{François Laplantine}

Volume 20, numéro 2, printemps 2008

Les musiques et la mort

URI : https://id.erudit.org/iderudit/018351ar

DOI : https://doi.org/10.7202/018351ar

Aller au sommaire du numéro

Éditeur(s)

Université du Québec à Montréal

ISSN

1180-3479 (imprimé)

1916-0976 (numérique)

Découvrir la revue

Citer cet article

Laplantine, F. (2008). Jouer et danser la tarentelle pour guérir la morsure de la tarentule Réflexions ethnopsychiatriques sur un " culte de la mort ».

Frontières, 20(2), 77-82. https://doi.org/10.7202/018351ar
Résumé de l'article

Cet article propose des réflexions ethno-psychiatriques sur l'un des derniers cultes de possession européens : le rituel thérapeutique de la tarentule, qui a été observé en Italie du Sud. La discussion présentée aborde entre autres l'efficacité du rite et la dimension esthétique des cérémonies cathartiques. 


\section{Résumé}

Cet article propose des réflexions ethnopsychiatriques sur l'un des derniers cultes de possession européens: le rituel thérapeutique de la tarentule, qui a été observé en Italie du Sud. La discussion présentée aborde entre autres l'efficacité du rite et la dimension esthétique des cérémonies cathartiques.

Mots clés: ethnopsychiatrie - rituel tarentelle - catharsis - religion pathologie.

\section{Abstract}

This article proposes ethnopsychiatric reflections on one of the last European possession cults: the therapeutic ritual of the tarantula found in southern Italy. The present discussion tackles among other things the efficiency of the rite and the aesthetic dimension of cathartic ceremonies.

Keywords: ethnopsychiatry - ritual tarantella - catharsis - religion pathology.

\section{Jouer et danser la tarentelle pour guérir la morsure de la tarentule Réflexions ethnopsychiatriques sur un «culte de la mort »"}

\author{
François Laplantine, \\ professeur, Département d'anthropologie, \\ Université Lumière Lyon II.
}

Les réflexions esquissées dans ce chapitre sont nées d'une proposition qui m'a été faite en 1977 par le photographe André Martin. Ce dernier m'a demandé de réagir en ethnologue aux photographies qu'il avait réalisées à partir du début des années 1960 en Italie du Sud et d'écrire la préface de son ouvrage Les noires vallées du repentir (2000). Ce sont en particulier les clichés des orchestres musicaux jouant et faisant danser la tarentelle afin de soigner la morsure de la tarentule qui ont retenu mon attention.

La dernière danse rituelle d'une femme qui avait été mordue par cette araignée venimeuse eut lieu à Galatina, le 29 mai 1993. Des scènes de la sorte dont il va être question maintenant, je ne les ai pas vues. Elles ont été médiatisées par des images et réinscrites dans leur contexte social, culturel, historique à partir de la lecture des ouvrages de l'anthropologue Ernesto de Martino (1999, 2000, 2001).
À Oppido Lucano, deux fois l'an, des femmes toutes habillées de noir viennent à dos de mulet implorer le pardon de la Madone. Elles s'accusent de fautes qu'elles n'ont pas nécessairement commises et se libèrent ainsi rituellement, par le biais de l'imaginaire, d'un passé de soumission et de servitude, vécu comme une épreuve que seul le sacré paraît pouvoir soigner, ou tout du moins aménager. Pour accentuer leur sentiment d'humiliation devant le surnaturel, elles se traînent, la langue collée sur le sol, jusqu'à la statue de la Vierge. Leurs visages reflètent la gravité, la douleur, la ferveur et le recueillement et en disent long sur le passé de misère des habitants de ces régions déshéritées.

À Palerme, pendant la fête de SainteRosalie, on pousse peut-être encore plus loin la dévotion et la soumission au sacré. La sainte protège de la maladie et l'on recueille l'eau de sa grotte. On se confesse, on s'accuse, on implore le pardon et le repentir. Génuflexions, prosternations, signes de croix, répétitions de prières, processions pieds nus autour du sanctuaire attestent 
à quel point l'existence quotidienne est vécue comme une punition. Et la panoplie d'ex-voto dont les catholiques italiens en particulier semblent si friands, les reliques, les bras et les jambes en cire sont autant de supports permettant de fixer la croyance pour redonner de la force et de l'espoir et plus précisément pour combattre la maladie. Cette familiarité des Italiens du Sud et des Siciliens avec le sacré s'exprime en particulier dans ce qu'André Martin appelle un «culte de la mort». Comme pour mieux apprivoiser cette dernière, montrer qu'elle est après tout une délivrance, on l'appelle au secours, on se rassemble à Naples même, dans ces nécropoles des premiers chrétiens, pour célébrer la puissance des crânes et des ossements. Bien évidemment, ces Italiens du Sud, pas plus que les Africains comme on l'a prétendu longtemps, n'adorent les ancêtres pour eux-mêmes, mais ils investissent de puissance tout ce qui leur apparaît comme autant de médiations entre l'homme et le sacré.

Il ne faudrait pas croire cependant que l'univers magico-religieux des habitants de Lucanie, de Calabre, des Pouilles et de Sicile est tout entier composé à base de deuil. Les Italiens du Sud savent faire la fête et savent la faire jusqu'au bout, et cette exaltation trépidante qui explose en suivant le rythme calendaire des cérémonies, comme à San Fratello en Sicile sur le parvis de l'église, est l'expression dramatique de la décharge émotionnelle et théâtrale du groupe tout entier. Les conflits individuels et collectifs sont représentés et joués symboliquement: le Bien et le Mal, Jésus et Judas, le Diable, le Juif et le Bon Dieu. Bien sûr, si l'on joue le Mystère de la Passion, c'est parce que la vie est vécue comme un martyre dont il faut se décharger, ensemble, en suivant les axes d'un dispositif rituel, et l'explosion festive, le temps du carnaval, contraste avec la monotonie des jours laborieux, où la pénitence et le sens du Carême finissent par reprendre le dessus. Il existe enfin un phénomène d'une rare intensité et d'une exceptionnelle violence: c'est le mythe et le rituel thérapeutique de la tarentule qui fut l'un des derniers cultes de possession européens.

Dans ce culte, au premier abord d'une singulière étrangeté, les femmes ne se contentent pas, comme tout à l'heure, de parcourir le sanctuaire à plat ventre: elles rampent sur le dos en poussant des cris, grimpent sur l'autel, et puisqu'elles sont devenues des tarentules, la foule des fidèles rassemblés pour la circonstance ne s'en approche qu'avec précaution, car, comme on dit à Galatina: "Elles sont dangereuses. »C'est sur ce phénomène paroxystique que je voudrais m'arrêter quelque peu, tant je suis frappé par la richesse et la luxuriance des symboles ici évoqués et mis en jeu.

Le mythe de la tarentule s'appuie comme tous les mythes sur un support réel, et d'abord sur le fait suivant: la morsure venimeuse d'une petite araignée qui s'appelle le latrodecte. Mais d'emblée l'imagination paysanne se met au travail; le latrodecte, dont la morsure engendre un syndrome toxique très grave, ne répond pas aux caractéristiques que les hommes et les femmes des Pouilles projettent sur ce qui leur apparaît monstrueux et redoutable. C'est une autre araignée, la «lycose tarentula », qui est parfaitement inoffensive, elle, mais qui a un aspect velu, une couleur sombre, une taille impressionnante et un comportement terriblement agressif l'été à l'époque de la ponte des œufs, qui se voit attribuée les caractères de la première. Si l'espèce zoologique «tarentule» se transforme en un symbole, celui d'une bête féroce qui agresse l'homme au moment des récoltes annuelles, c'est en raison d'une série d'images qui sont ressenties comme particulièrement puissantes et qui frappent l'inconscient villageois de la région de Galatina, donnant libre cours à l'imaginaire. Les tarentulés en crise jouent l'empoisonnement réel en modelant leurs symptômes sur le modèle fourni par la morsure du latrodecte. Mais à partir de là, le symbole de la tarentule s'étend, s'enrichit et forme la texture vivante d'un mythe d'une puissante intensité: le mythe de l'agression du "mauvais passé » qui, comme le dit Ernesto de Martino, «mord» et «remord» chaque année à la saison chaude où les paysans récoltent ce qu'ils ont semé.

La répétition saisonnière d'une morsure fictive où l'on mime une série de symptômes qui, eux, sont bien réels, le fait qu'à Galatina, le 29 juin de chaque année, les tarentulés affluent de toute la province pour se mettre sous la protection de l'Apôtre des gentils - qui lors de l'épisode de Malte avait maîtrisé le serpent venimeux -, le fait qu'il serait de fort mauvais goût de jouer à la tarentule dans ce bastion de saint Paul, qui jouit aux yeux des habitants de la région d'une immunité totale, ou hors saison, tout cela nous indique déjà les limites de l'approche proprement médicale du phénomène qui est d'emblée discipliné, canalisé, ordonné et façonné dans les cadres d'un dispositif liturgique dont nous allons maintenant parler.

L'animal mythique, qui «mord» et «remord» chaque année, est un animal coloré, sonore, chantant et dansant. Il faut donc pour en venir à bout faire appel à de petits orchestres ambulants composés de violons, de cithares et de tambourins qui joueront ces mélodies bien connues des Italiens, et qui précisément s'appellent les «tarentelles». Puisque la morsure est musicale, elle ne peut être évoquée qu'en jouant de la musique, puisque la tarentule se déplace en dansant la tarentelle, il faudra la lui faire danser. Enfin puisqu'elle aime les couleurs, on devra lui lancer toute une gamme de tissus, de rubans et d'étoffes. Le premier moment du rite consiste à engager un dialogue avec l'araignée, et pour cela il faudra trouver la note juste, l'air qui lui convient et la couleur qui est la sienne. Les musiciens, telle une véritable équipe thérapeutique au travail, sont les intermédiaires entre les individus - ce sont le plus souvent des femmes - possédés par l'araignée et le sacré. Ils interviewent la bête, posent ce qu'il faut bien appeler un diagnostic, qui entraînera à son tour un pronostic. Certaines tarentulées sont allergiques à certaines mélodies, à certains pas de danse et à certaines couleurs. D'autres, en revanche, dès que la note juste a été trouvée, éprouvent une irrésistible impulsion à danser la tarentelle. C'est à travers ce jeu alterné de sympathies et d'antipathies qu'explosent littéralement, au cours de ces performances chorégraphiques et musicales qui peuvent durer plusieurs jours, les conflits du possédé, et qu'ainsi s'expulsent sa souffrance et son angoisse. La tarentulée en léthargie se redresse brutalement sous le rythme de la tarentelle qui l'arrache à sa stupeur et l'achemine graduellement vers la frénésie. Les musiciens l'initient à l'esprit et au corps possesseur de la bête. Plus encore, c'est le corps de la tarentulée elle-même qui devient un corps musical. On se fait araignée avec l'araignée, on s'identifie, comme disent les psychanalystes, avec son agresseur, et l'on exprime sa fureur érotique dans une ardente union sexuelle

PUISQUE LA MORSURE EST MUSICALE, ELLE NE PEUT ÊTRE ÉVOQUÉE QU'EN JOUANT DE LA MUSIQUE, PUISQUE LA TARENTULE SE DÉPLACE EN DANSANT LA TARENTELLE, 


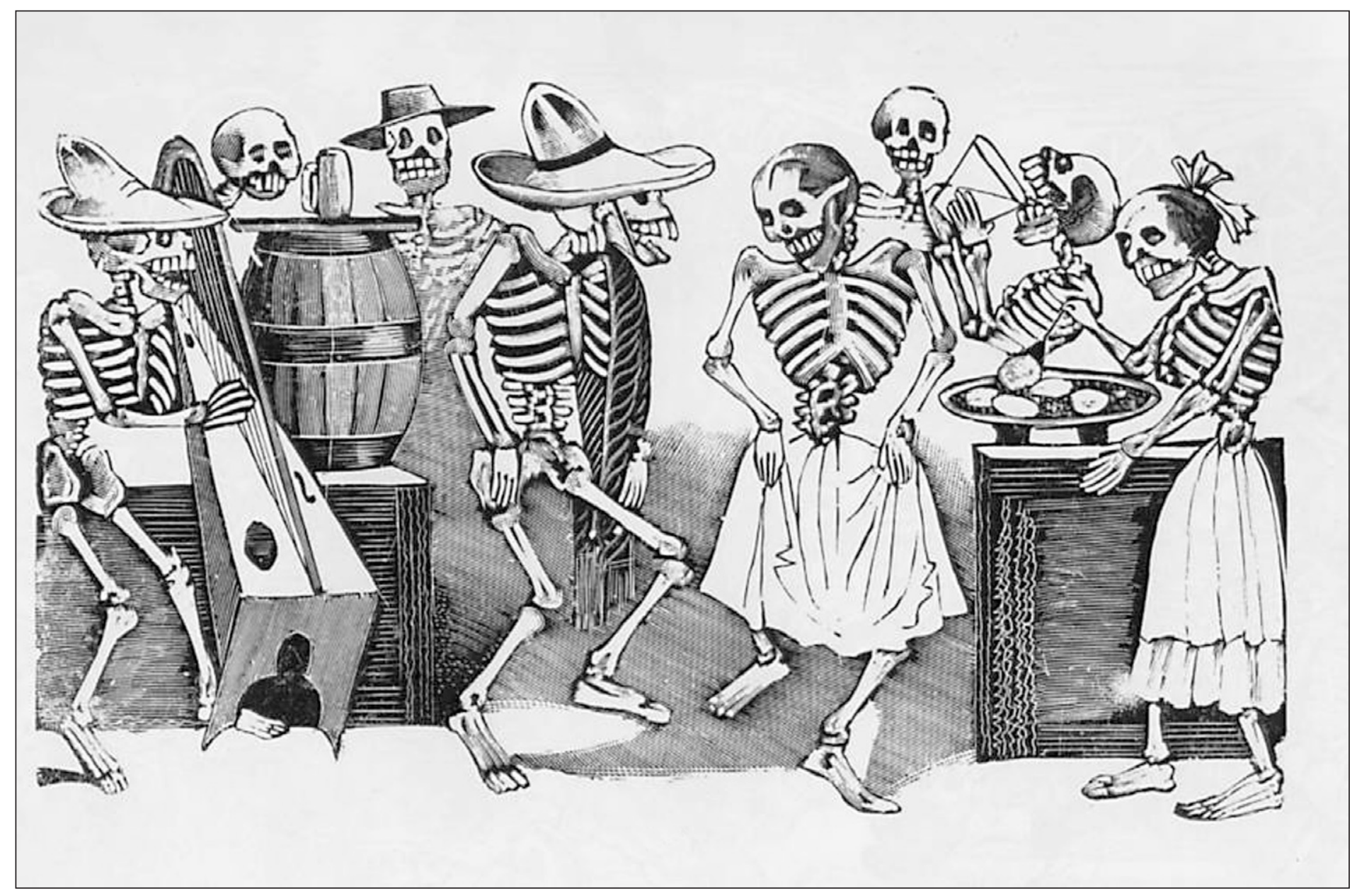

José Guadalupe POSADA (graveur mexicain) Gran fandango y francachela de todas las calaveras (Happy Dance and Wild Party of all the Skeletons) Gravure sur métal, Broadside, Mexico.

symbolique avec l'animal. La passion et la haine, le deuil et la mélancolie se fixeront selon les cas, sur telle mélodie ou sur telle étoffe colorée. Le vert, par exemple, parlera davantage aux individus frustrés par les amours perdues ou impossibles, et l'amour-propre bafoué par les interdits sociaux si vigoureux et si pesants en Italie $\mathrm{du}$ Sud se fixera, par exemple, sur les airs martiaux ou la couleur rouge. Enfin à cette phase du rituel - le temps de l'adorcisme et de l'initiation symbolique - succède le temps fort de l'exorcisme, le temps de ce que De Martino appelle très exactement la "dissociation agonistique», au cours duquel la tarentelle réussit comme on dit à faire «crever» la bête.

Ce rituel tout à fait exceptionnel dans l'Europe du $\mathrm{XX}^{\mathrm{e}}$ siècle - qui n'est pas sans évoquer certaines cérémonies des Aissaouas au Maroc - est fondé sur une série de stimulations sonores, chromatiques et chorégraphiques chargées d'induire un mythe (gonflé de la mémoire collective des paysans des Pouilles) qui transmet un message à l'inconscient. Par tout un jeu de correspondances symboliques d'une violence et d'une puissance intenses, la maladie circule entre l'araignée, les tarentulés, les musiciens, la famille et la communauté villageoise rassemblée pour la circonstance. Ces cérémonies permettent tout à la fois de débloquer les impulsions agressives qui «mordent» et «remordent» les possédés à la saison chaude et de libérer l'étreinte érotique interdite hors saison. Elles leur fournissent un horizon de résolution rituelle et de liquidation saisonnière des symptômes, un instrument efficace de décharge de venin et de tout ce qu'il représente, jusqu'au prochain été.

Je crois qu'il est possible, sans prétendre épuiser la richesse symbolique de ce phénomène, de repérer et de nommer deux groupes de significations que voici.

\section{LE PSYCHIATRE \\ DEVANT LA TARENTULE}

Par la brutalité des crises et par l'ensemble des manifestations d'agitation psychologique et rythmique, d'hallucinations auditives et visuelles qu'elle déclenche, la tarentule évoque irrésistiblement ce que la psychiatrie désigne depuis Kraepelin sous le nom de «bouffée délirante». En Afrique noire, l'individu qui présente le plus souvent un assez bon état général est soudainement agressé par des génies alternativement protecteurs et pathogènes qui l'envahissent littéralement. Ce qui semble être le cas également des tarentulés des Pouilles. La morsure venimeuse est une morsure mélodique par laquelle le mal, en l'occurrence la bête, pénètre la personnalité du malade, lui donne des ordres contre son gré, bref le possède. Mais la projection saisonnière des conflits sur l'animal mythique semble jouer également sur un autre registre des grands processus psychopathologiques: celui de la psychose maniacodépressive. L'angoisse dépressive a été décrite par Mélanie Klein comme la peur du bébé de perdre sa mère à tout jamais. C'est à l'époque où le sadisme oral est à son apogée que l'enfant est le plus menacé de se voir submergé par ses propres pulsions destructrices à l'égard de celle qui lui donne tout, ou plus précisément dont il a tout à attendre: affection, chaleur et nourriture.

Si la dépression, que la psychanalyse qualifie aussi de mélancolie, est un état comparable à la souffrance du deuil, la réaction maniaque elle, prend son contrepied exact: je vis dans la hantise de perdre l'être que j'aime le plus au monde, et bien je n'y pense plus, je fais la fête jusque et y compris dans mon corps en projetant l'image de ma toute-puissance. 
Je suis personnellement frappé par le fait que l'état des tarentulés passe alternativement du noir de la langueur à la fureur agressive qui, comme nous venons de le voir, est une fureur colorée, et que le rythme même de la tarentelle est d'abord lent, lancinant et lamentable au sens étymologique du terme, puis rapide et joyeux.

Ce venin, qui pénètre dans les veines et qui réactualise les conflits laissés sans solution, est un venin mélancolique qui provoque cette sensation de lassitude, de tristesse et de dépression anxieuse, si caractéristique des tarentulées qui se disent elles-mêmes "ennuyées » jusqu'à l'infini, comme si elles venaient de perdre l'objet de leur amour. Cet élément de souffrance, dû à la morsure fictive de la «mère-araignée », doit sans aucun doute être relié à la composante sadique/orale qui agit en chacun de nous, le plus souvent à notre insu. Mordu, l'individu éprouve un sentiment de tristesse incommensurable, puis sous l'effet musical du rythme alerte de la tarentelle, un besoin absolu de danser. Au deuil et à la mélancolie succèdent l'accès de fureur, le débordement et la libération érotique. Chaque année, le venin fait son effet, mais chaque année la musique reprend le dessus. Ainsi, il semble que d'un côté, l'angoisse dépressive saisonnière des tarentulées soit l'occasion d'évoquer la frustration quotidienne d'une existence laborieuse et pesante, mais de l'autre et lui succédant, nous trouvons la réalisation symbolique de tout ce que précisément l'ordre social réprime : le désir de puissance et d'amour.

C'est sur cet aspect délibérément festif des «carnavaletti » des femmes apuliennes, qui à la fois évoque l'agitation maniaque et s'en distingue, que je voudrais mettre l'accent maintenant.

Le rituel musical, chorégraphique et chromatique de la tarentule est à la fois la récapitulation de l'angoisse dépressive et l'envers de la dépression, c'est-à-dire d'une bonne part de la monotonie et de la difficulté de vivre en Italie du Sud, quand on est sans le sou et soumis à un ordre patriarcal rigide. D'un côté, les femmes jouent les amours interdites ou malheureuses, de l'autre, elles se livrent dans l'horizon cérémoniel qui est mis chaque été à leur disposition à ce qu'il faut bien appeler un passage à l'acte, même s'il est mimé sur le mode symbolique. On transforme ainsi son désespoir et sa lassitude en joie et en espérance, on dilapide en quelques jours tout ce que l'on possède pour payer ces petits orchestres, qui vont de maison en maison pour la réalisation de paradis éphémères, qui n'ont après tout rien d'artificiels.

\section{L'EXPLOSION RITUELLE DE LA MISĖRE}

Le caractère festif et liturgique du mythe de la tarentule en action, qui est à la fois une soupape de résolution et de défoulement des conflits individuels et collectifs et un régulateur de l'ordre social, nous montre à l'évidence les limites d'une interprétation exclusivement psychologique et psychiatrique, incapable à elle seule de rendre compte de la totalité d'un phénomène comme celui-ci.

Nul mieux que Danilo Dolci (1963) n'a décrit la grande misère de la Sicile et de l'Italie du Sud, le sous-développement, l'exploitation et le gaspillage de la terre et des hommes. Les tarentules se répartissent d'une manière préférentielle par famille, par sexe et par classe sociale. Il y a peu de chance que le grand bourgeois napolitain joue à la tarentule. En revanche, dans une société patriarcale qui étouffe la femme et interdit l'amour en dehors du mariage, la possession par l'araignée est une des solutions possibles mise à la disposition des jeunes filles en fleur condamnées à la continence sexuelle, des épouses recluses et des veuves, pour qu'elles se libèrent d'un passé d'exploitation, de soumission et de pauvreté. La première morsure coïncide le plus souvent avec la puberté, et ce sont les adolescentes, plus enchaînées que les jeunes gens par les exigences d'un ordre familial écrasant, qui explosent et se livrent aux étreintes amoureuses avec la bête, avec saint Paul, avec l'un et l'autre.

Ainsi, ce "retour du refoulé», qui explose au grand jour et est d'emblée reformulé dans des canaux sociaux acceptables par tout le monde, doit-il être mis en relation avec la condition sociale et économique des paysans d'Italie du Sud. Enfin, à la misère, à la ségrégation et à la faim s'ajoute la fatigue des récoltes. L'été apulien est une saison harassante. C'est l'époque où l'on s'expose aux coups d'un soleil brûlant. C'est aussi l'époque la plus dramatique de l'année, où l'on vit dans l'espoir du «pain» et du «vin», où l'on pourra régler ou non les dettes accumulées tout au long de l'année, et où se régleront aussi les comptes d'un passé qui a toujours été éprouvé, en Italie méridionale, un peu comme une malédiction et un destin inéluctable. Cette saison, où les villageois et les villageoises dépensent toutes leurs forces jusqu'aux limites de la résistance humaine, devient l'horizon symbolique d'évocation et d'expression du "mauvais passé». Ceux qui sont fragilisés par leur enfance et leur misérable condition sociale n'en peuvent plus, craquent alors littéralement et se mettent à jouer le comportement de l'agresseur. Or l'agresseur, ce n'est pas seulement l'araignée, c'est tout ce qu'elle désigne. Comme en Afrique noire, cette forme de possession méditerranéenne (issue des anciens cultes helléniques qui se sont laissés mouler et discipliner dans les cadres du catholicisme italien pour former une combinaison syncrétique pleinement originale) est l'un des instruments des classes opprimées, des femmes en particulier, qui récupèrent par ce biais un peu de puissance contre les couches dominantes.

\section{DES PRATIQUES PATHOLOGIQUES OU DES RIPOSTES THÉRAPEUTIQUES?}

Ces différentes lignes de forces, qui sont comme les grands nœuds de significations que les images d'André Martin permettent de repérer et de nommer, me conduisent à poser la question: ne nous trouvons-nous pas en présence d'un gigantesque délire collectif de villages entiers qui participent à la maladie ou à l'égarement de leurs membres? Autrement dit, les thèmes manifestement hallucinatoires, que manipulent ceux qui tombent malades, se prosternent, se frappent la poitrine et s'accusent d'un ordre social qu'ils subissent comme une punition et une fatalité, ne sont-ils pas identiques à la pensée populaire de l'Italie et de la Sicile traditionnelles? Ce qui revient à se demander: n'est-ce pas toute l'Italie du Sud qui serait folle?

Le problème posé est particulièrement délicat à dénouer, et pour ma part, je ne saurais trancher gordiennement sans apporter les nuances qui, je crois, s'imposent en matière de pathologie collective. D'une part, comme nous l'a appris Georges Devereux, nous ne pouvons nous placer simultanément du point de vue du psychologue et du psychiatre et du point de vue du sociologue ou de l'ethnologue. J'ajouterai, en ce qui me concerne, que même la complémentarité des différentes perspectives n'épuise pas, il s'en faut, la richesse mythique d'un phénomène comme la magie en Sicile ou la tarentule dans la province de Galatina. D'autre part, les systèmes prophylactiques et curatifs des Italiens du Sud, qui adhèrent massivement à leurs traditions magico-religieuses, m'apparaissent comme étant paradoxalement à la fois pathologiques et thérapeutiques. J'articulerai donc ainsi ce paradoxe.

1. Nous sommes en présence d'un univers de saints protecteurs, de forces surnaturelles et d'animaux mythiques qui semblent doubler systématiquement la réalité sociale et permettent de riposter du tac au tac aux calamités naturelles et aux malédictions qui s'abattent sur l'homme pécheur. Comment donc nommer cet univers psychologique qui tantôt, attribue tout 
L'ARAIGNÉE PÉNĖTRE DANS L'INCONSCIENT COMME UN SYMPTÔME SAISONNIER, ET EN RESSORT DIGÉRÉE ET RECRACHÉE PAR LE RITUEL THÉRAPEUTIQUE COMME UN MYTHE.

ce qui ne va pas à l'extérieur de l'individu et, tantôt, s'impute des fautes qu'il n'a pas commises? Comment nommer cette société qui apparaît incapable d'affronter ses propres difficultés sans interposer à tout moment un écran déformateur et ce système qui masque à l'individu ses propres conflits, et à la culture dont il fait partie, par un effet de distorsion perceptive, ses propres contradictions?

Ce qui relève manifestement de la pathologie, c'est à mon avis cette difficulté, pour ne pas dire cette impossibilité des Italiens du Sud qui font de leurs croyances magicoreligieuses une conviction inébranlable, à résoudre aussi au niveau politique le sous-développement, le gaspillage et les contradictions économiques et sociales. $\mathrm{Au}$ lieu de chercher des solutions qui existent dans la réalité, mais qui bien sûr ne peuvent être arrachées que par la révolte, les paysans opprimés de la botte italienne et de la Sicile ont recours à l'humiliation, à la mortification. Ce qui arrive est accepté comme une fatalité et une épreuve inéluctable qui sont attribuées, comme le dit De Martino, au «mauvais passé » qui accable les hommes et les femmes de ces régions.

Ainsi, l'interprétation que le malade donne de son délire ne fait que conduire jusqu'à ses ultimes conséquences l'interprétation globale que la société renvoie à elle-même. L'envoûtement, le mauvais sort, le mauvais œil qui vous torturent, la maladie et le malheur qui s'abattent sur vous ne font finalement que pousser jusqu'à leur aboutissement logique des modèles culturels qui sont le propre de tous. Et l'exorciste sicilien, de même que les prêtres qui font appel à tel ou tel saint protecteur, de même que les équipes musicales chargées d'évoquer puis d'écraser l'araignée ne font finalement rien d'autre que de confirmer et de ratifier un système partagé par tous: le cercle infernal du sorcier et du magicien. Ainsi, non seulement l'élaboration fantasmatique d'un délire individuel serait en tous points homologue aux systèmes de représentations mythiques en vigueur, mais plus encore, on deviendrait malade lorsque l'on adhérerait à fond et sans restriction aux normes de sa propre société. C'est le même système interprétatif qui explique la maladie, le mal et le malheur, et qui est utilisé pour les soigner ou les éviter. La magie italienne ne fonctionnerait-elle donc pas alors comme un gigantesque mécanisme social délirant de défense contre l'angoisse?

2. C'est ici, je crois, qu'il faut apporter quelques précisions qui indiqueront à la fois la légitimité mais aussi les limites de la compréhension psychologique et psychiatrique qui, poussée à l'excès au-delà de son aire de «rentabilité »(Devereux), finirait vite par fonctionner à vide et risquerait de devenir plus délirante encore que les phénomènes dont elle chercherait à rendre compte.

Je crois tout d'abord qu'il est difficile, pour ne pas dire impossible, de parler avec objectivité du délire et de la fonction hallucinatoire, dans une société qui depuis des siècles exorcise et pénalise nos facultés imaginaires et visionnaires. Une bonne part de la vie des peuples et de la vie des individus trouve aussi sa satisfaction ailleurs que dans ce que l'on nomme, non sans ambiguiité, le réel; et la capacité humaine de rêver et de vivre son rêve n'a vraiment rien de morbide. Il faut bien sûr, pour s'en apercevoir, accepter de sortir, par une mutation ethnologique du regard, de notre provincialisme culturel.

Si toute religion, comme toute création collective d'ailleurs, charrie inéluctablement une bonne part de délire, il ne s'ensuit nullement que toute la religion puisse être réduite au délire. Si nous reprenons le mythe de la tarentule, je dirais que s'il me semble parfaitement exact de poser que la part de bouffée délirante et d'angoisse maniaque et dépressive des tarentules est homogène à la culture magique de la population apulienne dans ses matériaux constitutifs. Mais il faut immédiatement ajouter que ces symptômes lui sont hétérogènes dans leur processus psychologique et leur économie interne. Ce qu'il conviendrait alors de montrer point par point, c'est que toutes les séquences culturelles et cultuelles du rite thérapeutique en action agissent aussi dans une direction qui, précisément, est contraire à celle de la maladie. Même si ce n'est que le temps d'un été, l'équipe de musiciens thérapeutes fournit à ceux et celles qui, "mordus», tombent malades, les significations qui leur font défaut. Elle remodèle, discipline et finalement canalise les crises dans un lieu, un temps et un horizon rituel qui feront jusqu'à l'année suivante cesser les symptômes. Alors que privé de mythe, le défoulement des paysans des Pouilles ne serait qu'une psychose individuelle, il trouve dans le cadre de la tarentule et de la tarentelle un instrument thérapeutique de résolution collective.

Les critères nosologiques implicites de ce que j'appellerais la psychiatrie rituelle de l'Italie du Sud ne sont évidemment pas les nôtres. Dans la province de Galatina, celui qui délire, ce n'est pas celui qui croit à la morsure venimeuse de l'araignée, c'est celui qui n'y croit pas. En revanche, tout dans notre culture du psy (psychologue, psychanalyste, psychiatre ) nous prédispose à comprendre un culte de possession comme celui de la tarentule à partir de la bouffée délirante, de l'hystérie ou de l'agitation maniaque, alors qu'il serait aussi parfaitement légitime de suivre le processus inverse: comprendre la bouffée délirante qui est un phénomène individuel à partir de la possession qui est un rituel collectif, et nous verrions alors que la première réalise un écart considérable par rapport à la seconde. La croyance aux saints protecteurs, aux animaux mythiques et aux «créatures de Saint-Paul» comme on dit à Galatina, consiste moins à halluciner le réel qu'à l'interpréter. Parler de Saint-Paul, de Sainte-Rosalie, de l'araignée, du scorpion, du serpent et de la Madone consiste à nommer des instances psychologiques et sociologiques d'identification individuelle et culturelle qui, après tout, en valent bien d'autres. Prenons garde à ce que la dérision ou la réduction desséchante dans les grilles préétablies de la «psycho quelque chose», ou de la "socio quelque chose», ne tourne pas à l'arrogance et au procès qu'une société ou une époque intenterait à une autre. Rendre compte des systèmes de représentations traditionnelles méditerranéennes, comme d'un espace fantasmatique et illusoire venant doubler d'une manière superflue le réel, serait de notre côté entretenir l'illusion - car c'en est une aussi - que l'on pourrait se placer du point de vue de Sirius, c'est-à-dire du point de vue d'un observateur lucide et impartial qui aurait, lui, toutes les caractéristiques que l'on attribue habituellement à la divinité.

La seule distinction pertinente que l'ethnologue et l'ethnopsychiatre puissent finalement retenir en questionnant les interprétations magico-religieuses de la société italienne méridionale, c'est celle du mythe et du symptôme. Si «délire» il y a, il remplit le corps de tous les participants du culte des saints d'un langage d'emblée culturel qui assure la communication entre les hommes. La crise d'agitation hallucinatoire aiguë agit, elle, dans un sens rigoureusement inverse : elle réorganise la personnalité en la désocialisant, en la désindividualisant et en l'appauvrissant. Elle est un échec et une grimace de la possession, elle est la réduction du mythe 
au symptôme, à travers lequel le psychiatre parisien, lyonnais, montréalais, romain ou napolitain n'a évidemment cette fois aucun mal à diagnostiquer le processus d'une pathologie conventionnelle qui, non dans son contenu, mais dans son économie, lui est familière. Alors que la décompensation hallucinatoire camoufle et révèle simultanément une angoisse individuelle qui est souvent une souffrance atroce, les pratiques culturelles traditionnelles de ces Italiens du Sud et de ces Siciliens sont la célébration fervente et enthousiaste d'un mythe collectif, c'est-à-dire d'une parole socialisée qui circule entre tous les participants.

Ainsi donc, si ces manifestations d'exaltation religieuse et de ferveur mystique connotent une économie psychique archaïque qui prédispose à une forme de psychose confusionnelle aiguë, il ne s'ensuit nullement que la société rurale de la botte italienne et de la Sicile ait été une société psychotique de part en part. Tout ce que l'on peut dire, c'est que lorsque les mécanismes de défense sublimatifs (constitués notamment par la croyance en la toute-puissance des saints) échouent, lorsqu'un individu donné n'arrive plus à réagir à une série de traumatismes, de frustrations et d'échecs autrement que d'une manière pathologique, il le fait selon le modèle projectif et brutal qu'on lui a appris et qu'on attend du reste de lui. Mais aussitôt, il trouve à sa portée un dispositif rituel de reflux. C'est toute l'ambivalence $\mathrm{du}$ «jeu» de la tarentule qui est simultanément une maladie et une réaction à la maladie. L'araignée pénètre dans l'inconscient comme un symptôme saisonnier, et en ressort digérée et recrachée par le rituel thérapeutique comme un mythe.

\section{L'EFFICACITÉ SYMBOLIQUE}

Il m'apparaît en fin de compte que les pratiques médico-magiques qui sont mises en scène en Italie méridionale et en Sicile, et qu'André Martin a si bien réussi à capter sur la pellicule, ne sauraient être réduites à leurs conditions psychologiques et sociologiques d'émergence. De même que les explications médicales du XVIII ${ }^{\mathrm{e}}$ siècle, qui se sont empressées de naturaliser ce qui relève de l'imaginaire, sont aujourd'hui frappées de caducité, j'ai personnellement la conviction que nos explications d'aujourd'hui en «psy» et en «socio» (dévalorisantes et cannibaliques en matière de mythologies), qui en disent à la fois trop et pas assez, risquent fort de subir le même sort avec le temps.

Dire, par exemple, que la tarentule est un désordre psychologique me semble parfaitement exact, et pourtant tellement insuffisant. Prétendre, d'autre part, qu'elle est une aliénation sociale est, sans doute, un point de vue tout aussi légitime, mais qui risque de passer à côté de l'essentiel, en confondant ce qui n'est qu'un facteur déclenchant - la pauvreté de l'Italie du Sud - avec la luxuriance d'un phénomène qui le déborde infiniment. Ce qui subsiste finalement, une fois que les systèmes de représentations magiques des Apuliens, des Calabrais et des Siciliens ont été passés au crible des analyses des chercheurs de différentes disciplines, c'est ce jeu dramatique et ce circuit fantastique (et pas seulement fantasmatique) du sort jeté et levé, de l'agression subie et retournée, du venin de la Bête et de l'esprit de l'Apôtre, de l'impuissance et de la fécondité, de la castration et de l'érotisme, de la vie et de la mort. Et c'est aussi l'autonomie relative mais bien réelle de l'efficacité symbolique de la musique et de la danse.

La morsure fictive puis la remorsure, le venin, l'animal mythique, la crise, la cure et saint Paul lui-même sont les différentes significations qui échappent certes à la logique rationaliste d'un Occident exorcistique à l'égard de l'imagination collective. Il n'empêche qu'ils forment entre eux une chaîne logique d'une cohérence et d'une précision redoutable, puisqu'elle est celle de l'inconscient. Si l'on se pose la question: de quoi les noces mystiques avec l'Apôtre, la danse de l'araignée ou le culte des reliques sont-ils les symboles? on pourra bien sûr répondre (selon la discipline scientifique dans laquelle on a été formé, mais aussi selon sa personnalité) : de l'aliénation sociale ou de la frustration. Mais il subsistera encore ce qui m'apparaît personnellement l'essentiel: le fait qu'il y a dans les pèlerinages à Galatina ou à Sainte-Rosalie, comme dans la reconstitution de la Passion à San Fratello, un résidu difficile à traduire dans les cadres de notre mentalité contemporaine. Ce sont certes des psychothérapies et des sociothérapies, mais aussi et surtout, beaucoup plus que de simples psychothérapies et de simples sociothérapies, inassimilables en tout cas aux techniques modernes de la réadaptation. Autrement dit, ces cérémonies cathartiques d'exaltation, qui s'efforcent de redonner un sens au désordre en le socialisant ont une dimension esthétique. Elles sont la célébration de rituels joués et symbolisés qui se déroulent à la limite du théâtre.

\section{Bibliographie}

DOLCI, D. (1963). Gaspillage, Paris, François Maspero.

\section{Note de la rédaction}

1. Cet article original fut soumis simultanément pour publication à Frontières et aux Éditions Beauchesne, où il figure au chapitre 8 de l'ouvrage Ethnopsychiatrie psychanalytique, paru en septembre 2007. 